INPLASY

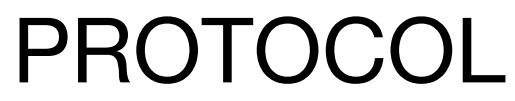

To cite: Ghosal et al. The cardiovascular benefits of GLP1-RA are directly related to their positive effect on glycaemic control: A metaregression analysis. Inplasy protocol 202210071. doi: 10.37766/inplasy2022.1.0071

Received: 15 January 2022

Published: 15 January 2022

Corresponding author: Samit Ghosal

amdasghosal@gmail.com

Author Affiliation:

Nightingale Hospital

Support: None.

Review Stage at time of this submission: Preliminary searches.

Conflicts of interest:

None declared.

\section{The cardiovascular benefits of GLP1- RA are directly related to their positive effect on glycaemic control: A meta-regression analysis}

Ghosal, S1; Sinha B2.

Review question / Objective: $\mathbf{P}$ (patient population) = Type 2 diabetes patients with high CV risk or established atherosclerotic cardiovascular disease; I (intervention) = Received drugs: GLP1-RA; C (control group) = Compared to a control group that received a placebo; $O$ (outcome) = Outcomes of interest included primary CV outcomes (MACE, CV death, MI, and Stroke).

Condition being studied: To explore whether the heterogeneity associated with the primary outcomes benefits can be attributed to the metabolic improvements associated with GLP1-RA. The plan is to use HBA1c, weight, and SBP reduction as moderators attempting to explain any variance between the true and observed effect size.

INPLASY registration number: This protocol was registered with the International Platform of Registered Systematic Review and Meta-Analysis Protocols (INPLASY) on 15 January 2022 and was last updated on 15 January 2022 (registration number INPLASY202210071).

\section{INTRODUCTION}

Review question / Objective: P (patient population) = Type 2 diabetes patients with high CV risk or established atherosclerotic cardiovascular disease; I (intervention) = Received drugs: GLP1-RA; C (control group) $=$ Compared to a control group that received a placebo; 0 (outcome) =
Outcomes of interest included primary CV outcomes (MACE, CV death, MI, and Stroke).

Rationale: To explore whether the heterogeneity associated with the primary outcomes benefits can be attributed to the metabolic improvements associated with GLP1-RA. The plan is to use HBA1c, 
weight, and SBP reduction as moderators attempting to explain any variance between the true and observed effect size.

Condition being studied: Type 2 Diabetes Mellitus and cardiovascular risk.

\section{METHODS}

Search strategy: We plan conducing this meta-regression analysis in concordance with the PRISMA guidelines. Electronic database search will be conducted using the Cochrane Library. Planned search keywords are "Glucagon like peptide 1 receptor agonist", "GLP 1RA", "liraglutide", "exenatide", "lixisenatide", "dulaglutide", "s e ma g l u t ide", "a I bi g l ut ide", "efpeglenatide", "MACE", "major advance cardiovascular events", "myocardial infarction" [MeSH], "AMI", "Stroke" [MeSH], "CV death", and "cardiovascular death". The drug-related and outcomes-related searches will be combined using the Boolean "OR", and both these entities will be combined using the Boolean "AND". Additional filters planned are RCT and date of selection between August 2008 (the publication of FDA guidance for industry) till date without any restriction on the language of reporting. Additional screening method planned is inclusion of studies having placebo in the comparator arm and MACE as the primary outcomes of interest.

Participant or population: The inclusion criteria included:- Randomized controlled trials.- Type 2 diabetes patients with an age limit of 18-75 years.- Placebo as the comparator arm.- Primary outcomes including 3-point MACE and a clear definition of its individual components.Reporting of the metabolic components addressed by GLP1-RA including HBA1C, weight, LDL-C, and systolic blood pressure.

Intervention: Intervention arm will include GLP1-RA group of drugs.

Comparator: Placebo.

Study designs to be included: Randomised controlled trials.
Eligibility criteria: The inclusion criteria included:- Randomized controlled trials.Type 2 diabetes patients with an age limit of 18-75 years.- Placebo as the comparator arm.- Primary outcomes including 3-point MACE and a clear definition of its individual components.- Reporting of the metabolic components addressed by GLP1-RA including HBA1C, weight, LDL-C, and systolic blood pressure.

Information sources: 1. An electronic database (the Cochrane Library) search will be conducted, preferably using MeSH terms to collect the citations of interest. 2. Additional google scholar search is planned to screen for any missing data from Cochran's search.

Main outcome(s): 1. Impact of HBA1c reduction on the cardiovascular benefits associated with GLP1-RA 2. Impact of HBA1c reduction by more than or equal to $0.9 \%$ (cut-off benefit according to UKPDS 35) and the CV benefits associated with GLP1-RA.

Additional outcome(s): Apart of influence of HBA1c we plan analyzing the impact of weight and SBP on the association of GLP1-RA and CV outcomes.

Quality assessment / Risk of bias analysis: Cochrane risk-of-bias algorithm.

Strategy of data synthesis: Dichotomous outcomes will be selected and analyzed. Participant data as reported in the individual randomized trials will be used to analyze effect size. 1. We are looking at including a minimum of 8 studies to perform the meta-analysis. This number is based on the historical data captured from previous meta-analyses involving GLP1-RA in T2D patients in CV outcomes trial. 2. Effect size variance will be calculated using Q statistics, $\mathbf{I}^{2}$, Tau, and $\mathbf{R}$-square values. 3. The significance of effect size will be based on the $95 \%$ confidence interval. 4 . In anticipation of heterogeneity across the included studies, we plan to choose the random effects model. BS and SG plan to extract the data for both primary (CV outcomes of interest), and secondary 
(HbA1c, weight, SBP) parameters. The chances of any error in entering the data would be cross-checked. All the selected citations would be evaluated along with their supplementary data and scored individually by BS \& SG. Any dispute would be reassessed, and a final decision would be taken by consensus.

Subgroup analysis: No subgroup analysis is planned.

Sensitivity analysis: No sensitivity analysis is planned.

Language: No restriction.

Country(ies) involved: India.

Keywords: GLP!_RA; Type 2 Diabetes; MACE; Meta-regression.

Contributions of each author:

Author 1 - Samit Ghosal.

Email: ramdasghosal@gmail.com

Author 2 - Binayak Sinha.

Email: binayak.sinha@gmail.com

BS and SG plan to extract the data for both primary (CV outcomes of interest), and secondary (HbA1c, weight, SBP) parameters. The chances of any error in entering the data would be cross-checked. All the selected citations would be evaluated along with their supplementary data and scored individually by BS. 